\title{
Digitalis Therapy and Risk of Recurrent Ventricular Tachyarrhythmias and ICD Therapies in Atrial Fibrillation and Heart Failure
}

\author{
Tobias Schupp ${ }^{\mathrm{a}} \quad$ Michael Behnes $^{\mathrm{a}}$ Christel Weiss $^{\mathrm{b}}$ Christoph Nienaber ${ }^{\mathrm{c}}$ Linda Reiser $^{\mathrm{a}}$ \\ Armin Bollow $^{\mathrm{a}}$ Gabriel Taton $^{\mathrm{a}}$ Thomas Reichelt ${ }^{\mathrm{a}}$ Dominik Ellguth ${ }^{\mathrm{a}}$ Niko Engelke $^{\mathrm{a}}$ \\ Jonas Rusnak $^{\mathrm{a}}$ Kathrin Weidner ${ }^{\mathrm{a}}$ Muharrem Akin ${ }^{d}$ Kambis Mashayekhi ${ }^{\mathrm{e}}$ Martin Borggrefe ${ }^{a}$ \\ Ibrahim Akin ${ }^{a}$ \\ ${ }^{a}$ First Department of Medicine, University Medical Center Mannheim (UMM), Faculty of Medicine Mannheim, \\ Heidelberg University, European Center for AngioScience (ECAS), and DZHK (German Center for Cardiovascular \\ Research) partner site Heidelberg/Mannheim, Mannheim, Germany; ${ }^{b}$ Institute of Biomathematics and Medical \\ Statistics, University Medical Center Mannheim (UMM), Faculty of Medicine Mannheim, Heidelberg University, \\ Mannheim, Germany; ${ }^{\mathrm{C}}$ Royal Brompton and Harefield Hospitals, NHS, London, UK; ${ }^{\mathrm{d}}$ Department of Cardiology and

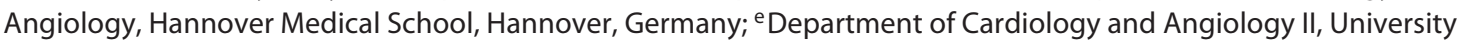 \\ Heart Center Freiburg, Bad Krozingen, Germany
}

\section{Keywords}

Ventricular tachycardia - Ventricular fibrillation .

Mortality · Digitalis · Heart failure · Atrial fibrillation ·

Implantable cardioverter defibrillator · Medical treatment ·

Pharmacological drugs

\begin{abstract}
Objective: This study sought to assess the impact of treatment with digitalis on recurrences of ventricular tachyarrhythmias in implantable cardioverter defibrillator (ICD) recipients with atrial fibrillation (AF) and heart failure (HF). Background: The data regarding outcomes of digitalis therapy in ICD recipients are limited. Methods: A large retrospective registry was used, including consecutive ICD recipients with episodes of ventricular tachyarrhythmia between 2002 and 2016. Patients treated with digitalis were compared to patients without digitalis treatment. The primary prognostic outcome was first recurrence of ventricular tachyarrhythmia
\end{abstract}

\section{KARGER}

(c) 2019 S. Karger AG, Basel

E-Mail karger@karger.com

www.karger.com/crd at 5 years. Kaplan-Meier and multivariable Cox regression analyses were applied. Results: A total of 394 ICD recipients with $\mathrm{AF}$ and/or HF was included (26\% with digitalis treatment and $74 \%$ without). Digitalis treatment was associated with decreased freedom from recurrent ventricular tachyarrhythmias (HR = 1.423; 95\% Cl 1.047-1.934; $p=0.023)$. Accordingly, digitalis treatment was associated with decreased freedom from appropriate ICD therapies (HR $=1.622 ; 95 \% \mathrm{CI}$ $1.166-2.256 ; p=0.004)$ and, moreover, higher rates of rehospitalization (38 vs. $21 \% ; p=0.001$ ) and all-cause mortality (33 vs. $20 \% ; p=0.011)$. Conclusion: Among ICD recipients suffering from $A F$ and $H F$, treatment with digitalis was associated with increased rates of recurrent ventricular tachyarrhythmias and ICD therapies. However, the endpoints may also have been driven by interactions between digitalis, AF, and HF.

(c) 2019 S. Karger AG, Basel

T. Schupp and M. Behnes contributed equally to this work. 


\section{Introduction}

Treatment with an implantable cardioverter defibrillator (ICD) is the therapeutic cornerstone of effective primary and secondary prevention of ventricular tachyarrhythmias and sudden cardiac death (SCD). ICDs have been shown to effectively decrease long-term mortality among patients suffering from cardiovascular diseases [1-3]. Additionally, optimal pharmacotherapy is recommended in order to ensure optimal long-term survival, especially with heart failure (HF) or multimorbid disease syndromes $[4,5]$.

The most common risk factor for the development of ventricular tachyarrhythmias is coronary artery disease, in more than $80 \%$ of affected patients [6]. Furthermore, the presence of atrial fibrillation (AF) has recently been shown to increase the risk of ventricular tachyarrhythmias and SCD [7-9]. The higher risk of ventricular tachyarrhythmias with AF may be attributed to an increasing burden of comorbidities including coronary artery disease and cardiomyopathies, or to higher rates of short-long-short cycles degenerating into ventricular tachyarrhythmias [9]. HF itself represents another independent risk factor for recurrent ventricular tachyarrhythmias, especially in ICD recipients for primary prevention [10]. Both AF and HF may be treated pharmacologically with cardiac glycosides in order to prevent recurrent episodes of supraventricular tachycardia and to potentially improve cardiac inotropy and HF-related symptoms $[11,12]$.

Whether there is a beneficial prognostic effect related to digitalis therapy is still under ongoing debate. Researchers question any effective reduction in rehospitalizations due to HF $[13,14]$ and mortality benefits for AF patients [15] and debate the heterogeneous results for long-term mortality among HF patients $[13,16]$. Accordingly, digitalis is no longer recommended as HF treatment, with prescriptions for this indication decreasing [17]. In contrast, the prospective randomized DIGIT-HF trial (EudraCT No. 2013-005326-38) currently reevaluates the prognostic impact of digitalis on systolic HF [17]. However, even fewer studies focused on the impact of digitalis on ICD-related therapies [1820].

Notably, there are no data with regard to whether digitalis affects recurrence of ventricular tachyarrhythmias in patients with a prior history of ventricular tachyarrhythmias. Therefore, this study evaluates the prognostic impact of digitalis therapy on the primary endpoint of recurrence of ventricular tachyarrhyth- mias, as well as on secondary endpoints (i.e., appropriate ICD therapies, rehospitalization, and all-cause mortality), in ICD recipients with HF, AF, and beta-blocker therapy surviving episodes of ventricular tachyarrhythmia.

\section{Subjects and Methods}

\section{Data Collection and Documentation}

The present study retrospectively included all consecutive ICD recipients surviving index episodes of ventricular tachyarrhythmia on admission between 2002 and 2016 at our institution. All relevant clinical data related to the index event, as well as those related to recurrences of ventricular tachyarrhythmias and rehospitalizations, were documented using the patients' files, daily records, documentation from diagnostic examinations and laboratory values, electrocardiograms (ECG), device recordings, and all further information derived from the electronic hospital information system.

Ventricular tachyarrhythmias comprised ventricular tachycardia (VT) and ventricular fibrillation (VF), as defined by current international guidelines $[4,21]$. Sustained VT was defined by a duration $>30 \mathrm{~s}$ or causing hemodynamic collapse within $30 \mathrm{~s}$, nonsustained VT by a duration $<30 \mathrm{~s}$ both with a wide QRS complex $(\geq 120$ $\mathrm{ms}$ ) at a rate $>100 \mathrm{bpm}$ [4]. Ventricular tachyarrhythmia at index was documented by 12-lead ECG, ECG telemonitoring, ICD, or, in case of an unstable course or during resuscitation, by external defibrillator monitoring. Documented VF was treated by ICD-related shock or external defibrillation, and in case of prolonged instability, with additional intravenous antiarrhythmic drugs during cardiopulmonary resuscitation. Electrical storm was defined as $\geq 3$ episodes of ventricular tachyarrhythmia requiring appropriate device therapy and occurring during a period of $24 \mathrm{~h}[4,22]$.

Further documented data contained baseline characteristics, prior medical history, prior medical treatment, length of index stay, detailed findings of laboratory values at baseline, and data derived from all noninvasive or invasive cardiac diagnostics and device therapies. These included coronary angiography, electrophysiological examination, and prior or newly implanted ICDs, pacemakers or cardiac contractility modulators which had already been implanted at index or at follow-up. Imaging modalities comprised echocardiography and cardiac magnetic resonance imaging.

The following device types were allowed: ICD, cardiac resynchronization therapy with defibrillator (CRT-D), and subcutaneous ICD. ICD recipients routinely presented to our clinic every 3-6 months for device check, as well as unscheduled in case of noticed device interrogations. Device setting and programming were performed according to current international guidelines by cardiologists specialized in electrophysiology during routine clinical care [4, $21,23]$. The device recordings were reevaluated retrospectively by independent cardiologists being blinded to the final data analysis.

The present study is derived from an analysis of the Registry of Malignant Arrhythmias and Sudden Cardiac Death - Influence of Diagnostics and Interventions (RACE-IT), a single-center registry including consecutive patients presenting with ventricular tachyarrhythmia and SCD being acutely admitted to the University Medical Center Mannheim (UMM), Germany (ClinicalTrials.gov identifier: NCT02982473), between 2002 and 2016. 


\section{Inclusion and Exclusion Criteria}

Consecutive ICD recipients with $\mathrm{HF}, \mathrm{AF}$, and beta-blocker therapy were included. All patients had a documented episode of ventricular tachyarrhythmia, which defines the index event. All patients analyzed had to survive index hospitalization and were discharged with documented beta-blocker therapy. HF was defined as a documented left ventricular ejection fraction (LVEF) $<45 \%$ [11]. Paroxysmal AF was defined as self-terminating in most cases within $48 \mathrm{~h}$ and, lately, for $\leq 7$ days, including AF episodes that are cardioverted within 7 days. Persistent AF lasts $>7$ days, including episodes terminated by cardioversion either with drugs or by direct current cardioversion after $\geq 7$ days. Permanent AF was defined as accepted by the patient and physician without pursuing further rhythm control. AF patients may have all degrees of LVEF [12].

The decision to treat patients with beta-blockers and digitalis was based on the discretion of the cardiologists during routine care according to European guidelines $[4,11,12,21]$. Patients without an activated ICD, HF, AF, beta-blocker treatment, or death at index hospitalization were excluded from the present analysis. To guarantee sufficient documentation of recurrent ventricular tachyarrhythmias, patients not presenting for at least one ICD check during follow-up were excluded from the present study.

\section{Definition of the Case and Control Groups}

The case group (digitalis group) comprised all patients with digitalis and beta-blocker treatment at discharge. Both digoxin and digitoxin were allowed. The control group (non-digitalis group) comprised all patients with beta-blocker but no digitalis treatment at discharge. All other medical therapies apart from beta-blockers and digitalis were allowed.

\section{Primary and Secondary Endpoints}

Follow-up period was set at 5 years for all outcomes. The primary prognostic endpoint was the first recurrence of ventricular tachyarrhythmia (VT or VF) as documented within the ICD protocols. Secondary endpoints were overall recurrences at follow-up, recurrences per patient, associated appropriate or inappropriate device therapies (first, overall, and per patient), first rehospitalization, and all-cause mortality at follow-up. Further stratification into subgroups of primary or secondary prevention and appropriate or inappropriate device therapies was performed.

Appropriate device therapy was defined as device-related therapy in the presence of VT or VF, including antitachycardia pacing (ATP), ICD shock, or both ATP and shock. Inappropriate device therapy was defined as ATP or ICD shock in the absence of VT or VF. First rehospitalizations comprised rehospitalizations due to $\mathrm{VT}, \mathrm{VF}$, acute myocardial infarction, acute HF, and inappropriate device therapy.

All-cause mortality was documented using our electronic hospital information system and by directly contacting state resident registration offices (bureau of mortality statistics) all across Germany. Identification of patients was verified by name, surname, day of birth, and registered living address.

\section{Statistical Methods}

Quantitative data are presented as mean \pm standard error of mean (SEM), median and interquartile range (IQR), and range depending on the distribution of the data, and they were compared using the Student $t$ test for normally distributed data or the MannWhitney $\mathrm{U}$ test for nonparametric data. Deviations from a Gauss-
Table 1. Distribution and dosages of digitalis and BB

\begin{tabular}{|c|c|c|c|c|c|}
\hline & \multicolumn{2}{|c|}{$\begin{array}{l}\text { No digitalis } \\
(n=290 ; 74 \%)\end{array}$} & \multicolumn{2}{|c|}{$\begin{array}{l}\text { Digitalis } \\
(n=104 ; 26 \%)\end{array}$} & \multirow{2}{*}{$\begin{array}{l}p \text { value } \\
\text { (distribution/ } \\
\text { dosage) }\end{array}$} \\
\hline & $\begin{array}{l}\text { Subjects, } \\
n(\%)\end{array}$ & $\begin{array}{l}\text { Dosage, } \\
\text { mg/day }\end{array}$ & $\begin{array}{l}\text { Subjects, } \\
n(\%)\end{array}$ & $\begin{array}{l}\text { Dosage, } \\
\mathrm{mg} / \text { day }\end{array}$ & \\
\hline Digoxin & - & - & $71(68)$ & $0.14 \pm 0.007$ & - \\
\hline Digitoxin & - & - & $33(32)$ & $0.08 \pm 0.003$ & - \\
\hline Metoprolol & $168(58)$ & $80.6 \pm 3.1$ & $43(41)$ & $73.4 \pm 4.7$ & $\mathbf{0 . 0 0 4} / 0.279$ \\
\hline Carvedilol & $72(25)$ & $19.5 \pm 1.6$ & $39(38)$ & $20.6 \pm 2.5$ & $\mathbf{0 . 0 1 4} / 0.706$ \\
\hline Bisoprolol & $33(11)$ & $5.8 \pm 0.5$ & $10(10)$ & $5.0 \pm 1.0$ & $0.693 / 0.470$ \\
\hline $\begin{array}{c}\text { Other type } \\
\text { of } B B\end{array}$ & $17(6)$ & & $12(12)$ & & 0.057 \\
\hline
\end{tabular}

Dosages are given as mean \pm SEM. Bold denotes significance. BB, betablocker.

ian distribution were tested by the Kolmogorov-Smirnov test. Spearman's rank correlation for nonparametric data was used to test univariate correlations. Qualitative data are presented as absolute and relative frequencies, and compared using the $\chi^{2}$ test or the Fisher exact test, as appropriate.

Firstly, the univariable Kaplan-Meier method was applied to evaluate prognostic differences within the entire cohort, between the subgroups of primary and secondary prevention, and between the subgroups of appropriate and inappropriate ICD therapies, each stratified according to digitalis and non-digitalis therapy. Then, the impact of digitalis was analyzed separately for AF and $\mathrm{HF}$, allowing the combination of AF and HF. Finally, the subgroups of "AF only" (AF patients without $\mathrm{HF}$ ) and "HF only" (HF patients without AF) were analyzed. Hazard ratios (HRs) are given together with $95 \%$ confidence intervals (CIs).

Secondly, multivariable Cox regression models were developed using the "forward selection" option, where only statistically significant variables $(p<0.05)$ were included and analyzed simultaneously. Predefined variables being used for multivariable Cox regressions included: baseline parameters (age, gender), chronic diseases (chronic kidney disease, diabetes mellitus), the combination of $\mathrm{AF}$ and $\mathrm{HF}$, and digitalis therapy. Patients without complete follow-up were censored (accepted lost-to-follow-up rate: $<10 \%$ ).

The result of a statistical test was considered significant with $p<0.05$, a statistical trend was defined as $p<0.10$. SAS, release 9.4 (SAS Institute Inc., Cary, NC, USA) and SPSS (version 25; IBM, Armonk, NY, USA) were used for statistical analysis.

\section{Results}

\section{Study Population}

A total of 394 consecutive ICD recipients with $\mathrm{HF}$ (87\%) and/or AF (44\%), of which $31 \%$ revealed both entities, was included. All patients survived an index episode of ventricular tachyarrhythmia and were discharged with 
Table 2. Baseline characteristics and comorbidities

\begin{tabular}{|c|c|c|c|}
\hline Characteristic & $\begin{array}{l}\text { Non-digitalis } \\
(n=290 ; 74 \%)\end{array}$ & $\begin{array}{l}\text { Digitalis } \\
(n=104 ; 26 \%)\end{array}$ & $p$ value \\
\hline Median age (range), years & $66(32-87)$ & $68(32-80)$ & 0.143 \\
\hline Male gender, $n(\%)$ & $239(82)$ & $83(80)$ & 0.555 \\
\hline Ventricular tachyarrhythmia at index, $n(\%)$ & & & 0.588 \\
\hline Ventricular tachycardia & $224(77)$ & $83(80)$ & \\
\hline Ventricular fibrillation & $66(23)$ & $21(20)$ & \\
\hline \multicolumn{4}{|l|}{ Cardiovascular risk factors, $n(\%)$} \\
\hline Arterial hypertension & $193(67)$ & $71(68)$ & 0.749 \\
\hline Diabetes mellitus & $76(26)$ & $33(32)$ & 0.280 \\
\hline Hyperlipidemia & $130(45)$ & $50(48)$ & 0.568 \\
\hline Smoking & $91(31)$ & $27(26)$ & 0.301 \\
\hline Cardiac family history & $41(14)$ & $16(15)$ & 0.757 \\
\hline \multicolumn{4}{|l|}{ Comorbidities at index stay, $n(\%)$} \\
\hline Heart failure & $252(87)$ & $91(88)$ & 0.875 \\
\hline Atrial fibrillation & $113(39)$ & $59(57)$ & 0.002 \\
\hline Paroxysmal & $76(26)$ & $41(39)$ & \\
\hline Persistent & $14(5)$ & $6(6)$ & 0.018 \\
\hline Permanent & $23(8)$ & $12(12)$ & \\
\hline Coronary artery disease & $225(78)$ & $71(68)$ & 0.059 \\
\hline Cardiogenic shock & $22(8)$ & $11(11)$ & 0.345 \\
\hline $\mathrm{CPR}$ & $55(19)$ & $14(14)$ & 0.333 \\
\hline Chronic kidney disease & $129(45)$ & $49(48)$ & 0.627 \\
\hline \multicolumn{4}{|l|}{ Medication at discharge, $n(\%)$} \\
\hline Beta-blocker & $290(100)$ & $104(100)$ & 1.000 \\
\hline ACE inhibitor & $221(76)$ & $75(72)$ & 0.408 \\
\hline $\mathrm{ARB}$ & $31(11)$ & $20(19)$ & 0.030 \\
\hline Aldosterone antagonist & $53(18)$ & $27(26)$ & 0.095 \\
\hline Amiodarone & $70(24)$ & $20(19)$ & 0.306 \\
\hline \multicolumn{4}{|l|}{ Mean ECG intervals \pm SD } \\
\hline PQ & $173 \pm 5$ & $190 \pm 9$ & 0.079 \\
\hline QRS & $117 \pm 5$ & $109 \pm 8$ & 0.391 \\
\hline QT & $415 \pm 5$ & $401 \pm 7$ & 0.138 \\
\hline LVEF, $n(\%)$ & & & 0.162 \\
\hline$\geq 55 \%$ & $18(6)$ & $5(5)$ & \\
\hline $54-45 \%$ & $11(4)$ & $1(1)$ & \\
\hline $44-35 \%$ & $79(28)$ & $20(21)$ & \\
\hline$<35 \%$ & $173(62)$ & $71(73)$ & \\
\hline Not documented & $9(-)$ & $7(-)$ & \\
\hline
\end{tabular}

Bold denotes significance. CPR, cardiopulmonary resuscitation; ACE, angiotensin-converting enzyme; ARB, angiotensin receptor blocker; ECG, electrocardiogram; LVEF, left ventricular ejection fraction.

or without digitalis treatment (non-digitalis $74 \%$ vs. digitalis $26 \% ; p=0.0001$ ). The target dosages were reached already at discharge, as seen for digoxin in $68 \%$ (mean dosage $0.14 \mathrm{mg} /$ day) and for digitoxin in $32 \%$ (mean dosage $0.08 \mathrm{mg}$ /day). Furthermore, the most commonly administered beta-blocker was metoprolol, with the target dosages being reached at discharge already (Table 1 ).

As seen in Table 2, the median age was 67 years, and most patients were of male gender (81\%). VT was more common than VF (77-80 vs. $20-23 \%$ ) at index in both treatment groups. The rates of VT and VF were comparable between the digitalis and the non-digitalis patients (VT: 77-80\%; VF: 20-23\%). The overall rates of HF were comparable between the digitalis and the nondigitalis group ( 88 vs. $87 \%$ ), whereas significantly more digitalis patients suffered from AF (57 vs. $39 \%$ ) with or without HF. The percentage of these patients with both entities was 26\% (non-digitalis) and 44\% (digitalis), re- 
Table 3. Baseline characteristics and comorbidities stratified by atrial fibrillation and heart failure

\begin{tabular}{|c|c|c|c|c|c|c|}
\hline \multirow[t]{2}{*}{ Characteristic } & \multicolumn{3}{|c|}{ Atrial fibrillation } & \multicolumn{3}{|l|}{ Heart failure } \\
\hline & $\begin{array}{l}\text { non-digitalis } \\
(n=113 ; 66 \%)\end{array}$ & $\begin{array}{l}\text { digitalis } \\
(n=59 ; 34 \%)\end{array}$ & $\begin{array}{l}p \\
\text { value }\end{array}$ & $\begin{array}{l}\text { non-digitalis } \\
(n=252 ; 73 \%)\end{array}$ & $\begin{array}{l}\text { digitalis } \\
(n=91 ; 27 \%)\end{array}$ & $\begin{array}{l}p \\
\text { value }\end{array}$ \\
\hline Median age (range), years & $70(32-87)$ & $68(45-80)$ & 0.717 & $66(22-87)$ & $68(32-82)$ & 0.079 \\
\hline Male gender, $n(\%)$ & $93(82)$ & $48(81)$ & 0.878 & $208(83)$ & $74(81)$ & 0.794 \\
\hline Ventricular tachyarrhythmia at index, $n(\%)$ & & & 0.301 & & & 0.741 \\
\hline \multicolumn{7}{|l|}{ Cardiovascular risk factors, $n(\%)$} \\
\hline Arterial hypertension & $78(69)$ & $43(73)$ & 0.599 & $164(65)$ & $62(68)$ & 0.599 \\
\hline Diabetes mellitus & $28(25)$ & $20(34)$ & 0.206 & $66(26)$ & $26(29)$ & 0.660 \\
\hline Hyperlipidemia & $49(43)$ & $31(53)$ & 0.252 & $114(45)$ & $45(50)$ & 0.490 \\
\hline Smoking & $29(26)$ & $15(25)$ & 0.973 & $83(33)$ & $21(23)$ & 0.079 \\
\hline Paroxysmal & $76(68)$ & $41(70)$ & & $50(20)$ & $30(33)$ & \\
\hline Persistent & $14(12)$ & $6(10)$ & 0.908 & $10(4)$ & $5(6)$ & 0.004 \\
\hline Permanent & $23(20)$ & $12(20)$ & & $15(6)$ & $11(12)$ & \\
\hline Coronary artery disease & $86(76)$ & $38(64)$ & 0.104 & $200(79)$ & $63(69)$ & 0.050 \\
\hline Cardiogenic shock & $8(7)$ & $8(14)$ & 0.165 & $20(8)$ & $9(10)$ & 0.566 \\
\hline $\mathrm{CPR}$ & $21(19)$ & $10(17)$ & 0.791 & $46(18)$ & $11(12)$ & 0.176 \\
\hline Chronic kidney disease & $55(49)$ & $27(46)$ & 0.717 & $115(46)$ & $43(48)$ & 0.772 \\
\hline \multicolumn{7}{|l|}{ Medication at discharge, $n(\%)$} \\
\hline Beta-blocker & $113(100)$ & $59(100)$ & 1.000 & $252(100)$ & $91(100)$ & 1.000 \\
\hline ACE inhibitor & $83(74)$ & $40(68)$ & 0.435 & $195(77)$ & $67(74)$ & 0.470 \\
\hline $\mathrm{ARB}$ & $11(10)$ & $12(21)$ & 0.055 & $27(11)$ & $19(21)$ & 0.017 \\
\hline Aldosterone antagonist & $25(22)$ & $17(29)$ & 0.332 & $50(20)$ & $24(26)$ & 0.194 \\
\hline $44-35 \%$ & $17(16)$ & $10(19)$ & & $79(31)$ & $20(22)$ & \\
\hline$<35 \%$ & $58(56)$ & $36(69)$ & & $173(69)$ & $71(78)$ & \\
\hline Not documented & $9(-)$ & $7(-)$ & - & $-(-)$ & $-(-)$ & - \\
\hline
\end{tabular}

ECG intervals are given as mean \pm SEM. Bold denotes significance. CPR, cardiopulmonary resuscitation; ACE, angiotensin-converting enzyme; ARB, angiotensin receptor blocker; ECG, electrocardiogram; LVEF, left ventricular ejection fraction.

spectively. Despite a slightly higher rate of angiotensin receptor blocker use in the digitalis group, no further significant differences were observed between the two groups, especially regarding rates of cardiogenic shock, cardiopulmonary resuscitation, and chronic kidney disease. Especially ECG intervals, coronary artery disease rates, and the LVEF were similar in both groups (Table 1).

Impact of Digitalis on Recurrent Ventricular Tachyarrhythmias
Table 3 presents the characteristics of patients with AF (left columns) and HF (right columns). Among the AF patients, comorbidities and LVEF groups were equally distributed. However, a higher rate of amiodarone treatment was seen for those patients not on digitalis. Among the HF patients, AF was more common in the digitalis group than in the non-digitalis patients, with a higher rate of angiotensin receptor blocker treatment. 


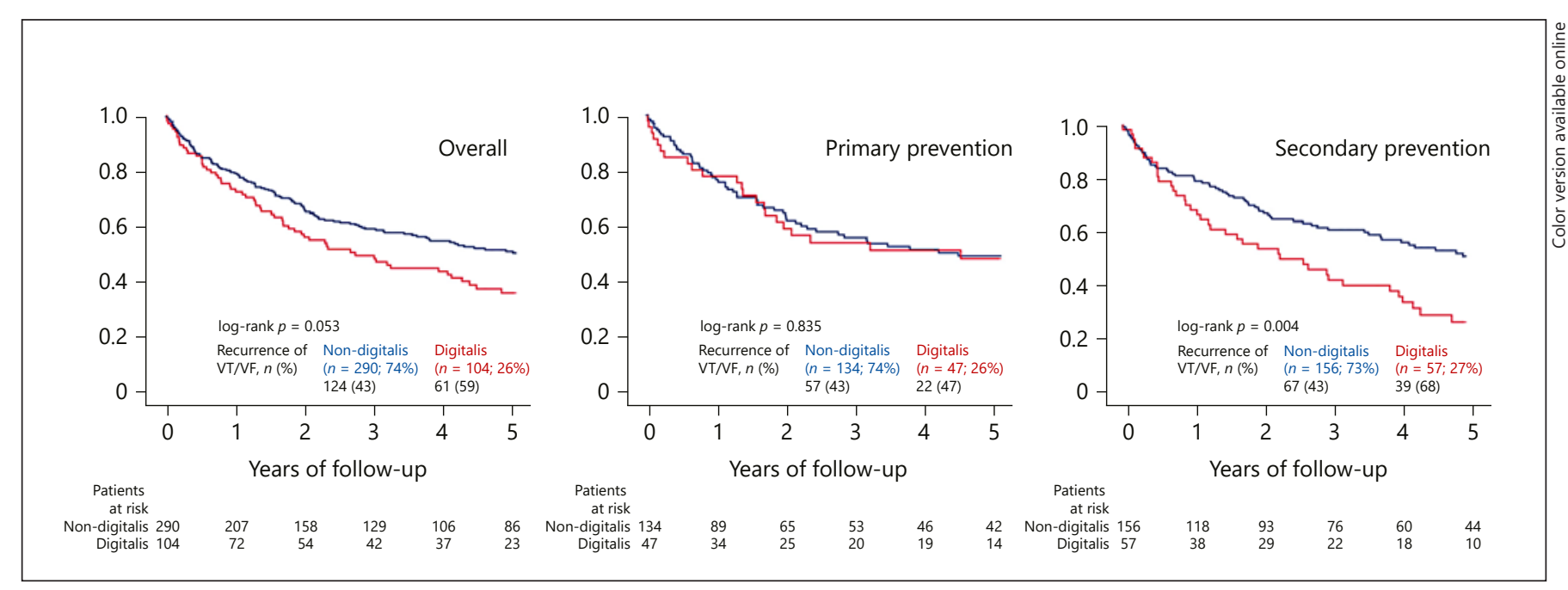

Fig. 1. Freedom from first recurrence of ventricular tachyarrhythmia (overall, left panel), stratified by primary (middle panel) and secondary preventive implantable cardioverter defibrillator recipients (right panel). VT, ventricular tachycardia; VF, ventricular fibrillation.

Fig. 2. Digitalis treatment decreased freedom from appropriate device therapy (left panel), but it did not affect freedom from inappropriate device therapy (right panel).

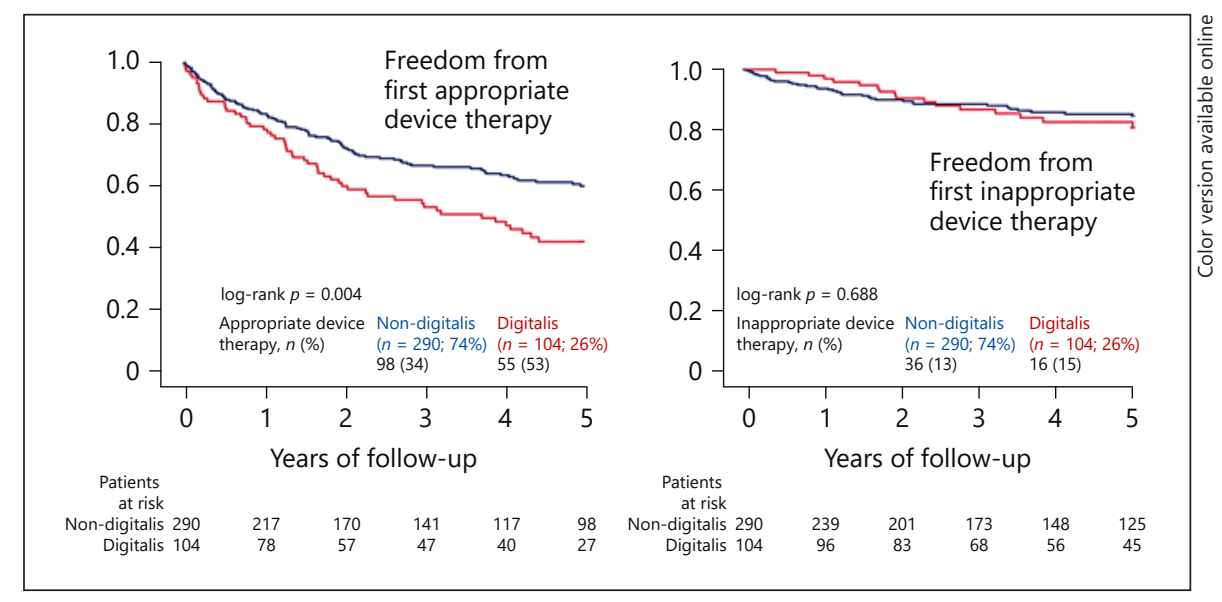

Table 4 outlines the ICD-related data for the study population. Most patients had an activated transvenous ICD (88-93\%), whereas a minority of the patients had a transvenous CRT-D or subcutaneous ICD (1-10\%). The ICD was mostly implanted for secondary prevention (55 vs. $45 \%)$. The median detection thresholds for VT (171 vs. $176 \mathrm{bpm}$ ) and for VF (214 bpm) were comparable between the two groups, as well as was the median cycle length of VT, ranging from 315 to $320 \mathrm{~ms}$ (Table 4).

\section{Follow-Up Data and Primary and \\ Secondary Endpoints}

At least $90 \%$ of the patients were followed up regularly within the follow-up period of 5 years ( 1,825 days) with $\geq 1$ ICD check-up every 6-12 months.
Freedom from first episodes of ventricular tachyarrhythmia was decreased in digitalis patients (59 vs. 43\%; $\log$-rank $p=0.023 ; \mathrm{HR}=1.423 ; 95 \%$ CI 1.047-1.934; $p=$ 0.023 ) (Table 4; Fig. 1, left panel). Notably, this difference was mainly observed among patients with secondary preventive ICDs ( 68 vs. $43 \%$; log-rank $p=0.004 ; \mathrm{HR}=1.776$; $95 \%$ CI $1.196-2.638 ; p=0.004)$, but not in primary preventive ICD recipients $(\log -\operatorname{rank} p=0.835)$ (Fig. 1, middle and right panels). The increasing rates of first recurrence were mainly attributable to sustained VT (43 vs. $25 \%$ ), which was also reflected in an increasing rate of overall sustained VT (53 vs. 31\%) (Table 4).

Freedom from first appropriate device therapy was decreased in digitalis patients ( 53 vs. $34 \%$; $\log$-rank $p=0.004$; $\mathrm{HR}=1.622 ; 95 \%$ CI 1.166-2.256; $p=0.004)$ (Fig. 2, left 
Table 4. ICD data and primary and secondary endpoints

\begin{tabular}{|c|c|c|c|}
\hline Characteristic & $\begin{array}{l}\text { Non-digitalis } \\
(n=290 ; 74 \%)\end{array}$ & $\begin{array}{l}\text { Digitalis } \\
(n=104 ; 26 \%)\end{array}$ & $p$ value \\
\hline Type of ICD, $n(\%)$ & & & 0.304 \\
\hline ICD & $255(88)$ & $97(93)$ & \\
\hline CRT-D & $28(10)$ & $6(6)$ & \\
\hline s-ICD & $7(2)$ & $1(1)$ & \\
\hline Implant indication, $n(\%)$ & & & 0.859 \\
\hline Primary prevention & $134(46)$ & $47(45)$ & \\
\hline Secondary prevention & $156(54)$ & $57(55)$ & \\
\hline \multicolumn{4}{|l|}{ ICD programming, bpm ${ }^{1}$} \\
\hline VT detection threshold & $171(167-171)$ & $176(167-176)$ & 0.073 \\
\hline VF detection threshold & $214(214-217)$ & $214(214-222)$ & 0.078 \\
\hline \multicolumn{4}{|c|}{ Primary endpoint } \\
\hline \multicolumn{4}{|c|}{ First recurrence of ventricular tachyarrhythmia, $n(\%)$} \\
\hline Overall & $124(43)$ & $61(59)$ & 0.006 \\
\hline Nonsustained VT & $33(11)$ & $9(9)$ & 0.440 \\
\hline Sustained VT & $73(25)$ & $45(43)$ & 0.001 \\
\hline VF & $18(6)$ & $7(7)$ & 0.570 \\
\hline Electrical storm & $18(6)$ & $11(11)$ & 0.143 \\
\hline \multicolumn{4}{|l|}{ Secondary endpoints } \\
\hline \multicolumn{4}{|l|}{ Overall recurrences at follow-up, $n(\%)$} \\
\hline Nonsustained VT & $53(18)$ & $19(18)$ & 0.999 \\
\hline Sustained VT & $90(31)$ & $55(53)$ & 0.001 \\
\hline VF & $28(10)$ & $14(14)$ & 0.281 \\
\hline Electrical storm & $18(6)$ & $11(11)$ & 0.143 \\
\hline VT cycle length, $\mathrm{ms}^{1}$ & $315(280-345)$ & $320(300-350)$ & 0.444 \\
\hline \multicolumn{4}{|l|}{ Recurrences per patient ${ }^{2}$} \\
\hline Nonsustained VT & $5.3 \pm 2.3$ & $8.4 \pm 4.8$ & 0.523 \\
\hline Sustained VT & $5.3 \pm 1.3$ & $9.2 \pm 3.6$ & 0.198 \\
\hline VF & $0.3 \pm 0.1$ & $0.4 \pm 0.2$ & 0.604 \\
\hline Electrical storm & $0.1 \pm 0.0$ & $0.1 \pm 0.0$ & 0.144 \\
\hline \multicolumn{4}{|l|}{ First device therapies, $n(\%)$} \\
\hline Overall appropriate device therapy & $98(34)$ & $55(53)$ & 0.001 \\
\hline Appropriate shock & $38(13)$ & $19(18)$ & 0.199 \\
\hline Appropriate ATP only & $53(18)$ & $32(31)$ & 0.007 \\
\hline Inappropriate device therapy & $36(13)$ & $16(15)$ & 0.450 \\
\hline \multicolumn{4}{|c|}{ Overall device therapies at follow-up, $n(\%)$} \\
\hline Overall appropriate device therapy & $98(34)$ & $55(53)$ & 0.001 \\
\hline Appropriate shock & $57(20)$ & $20(29)$ & 0.055 \\
\hline Appropriate ATP only & $80(28)$ & $44(42)$ & 0.006 \\
\hline Inappropriate device therapy & $36(13)$ & $16(15)$ & 0.450 \\
\hline \multicolumn{4}{|l|}{ Device therapies per patient ${ }^{2}$} \\
\hline Appropriate shock & $0.5 \pm 0.1$ & $1.5 \pm 0.5$ & 0.003 \\
\hline Appropriate ATP only & $4.4 \pm 1.1$ & $7.3 \pm 3.5$ & 0.283 \\
\hline Inappropriate device therapy & $0.4 \pm 0.1$ & $0.4 \pm 0.1$ & 0.608 \\
\hline \multicolumn{4}{|l|}{ First rehospitalization, $n(\%)$} \\
\hline Overall & $61(21)$ & $39(38)$ & 0.001 \\
\hline VT & $24(8)$ & $17(16)$ & 0.021 \\
\hline VF & $5(2)$ & $1(1)$ & 1.000 \\
\hline AMI & $5(2)$ & $1(1)$ & 1.000 \\
\hline Acute heart failure & $16(6)$ & $14(13)$ & 0.009 \\
\hline Inappropriate device therapy & $11(4)$ & $6(6)$ & 0.395 \\
\hline All-cause mortality at 5 years, $n(\%)$ & $59(20)$ & $34(33)$ & 0.011 \\
\hline
\end{tabular}

Bold denotes significance. ICD, implantable cardioverter defibrillator; CRT-D, cardiac resynchronization therapy with defibrillator; s-ICD, subcutaneous ICD; AMI, acute myocardial infarction; ATP, antitachycardia pacing; HF, heart failure; VF, ventricular fibrillation; VT, ventricular tachycardia. ${ }^{1}$ Median (IQR). ${ }^{2}$ Mean \pm SEM. 
Fig. 3. a AF patients. Digitalis therapy was still associated with a trend for an increased risk of recurrent VT/VF (left) and appropriate ICD therapy (right) in the subgroup of AF patients. b HF patients. Digitalis therapy was not associated with an increased risk of recurrent VT/VF (left), but with an increased risk of appropriate ICD therapy (right) in the subgroup of HF patients. AF, atrial fibrillation; HF, heart failure; VT, ventricular tachycardia; VF, ventricular fibrillation; ICD, implantable cardioverter defibrillator.

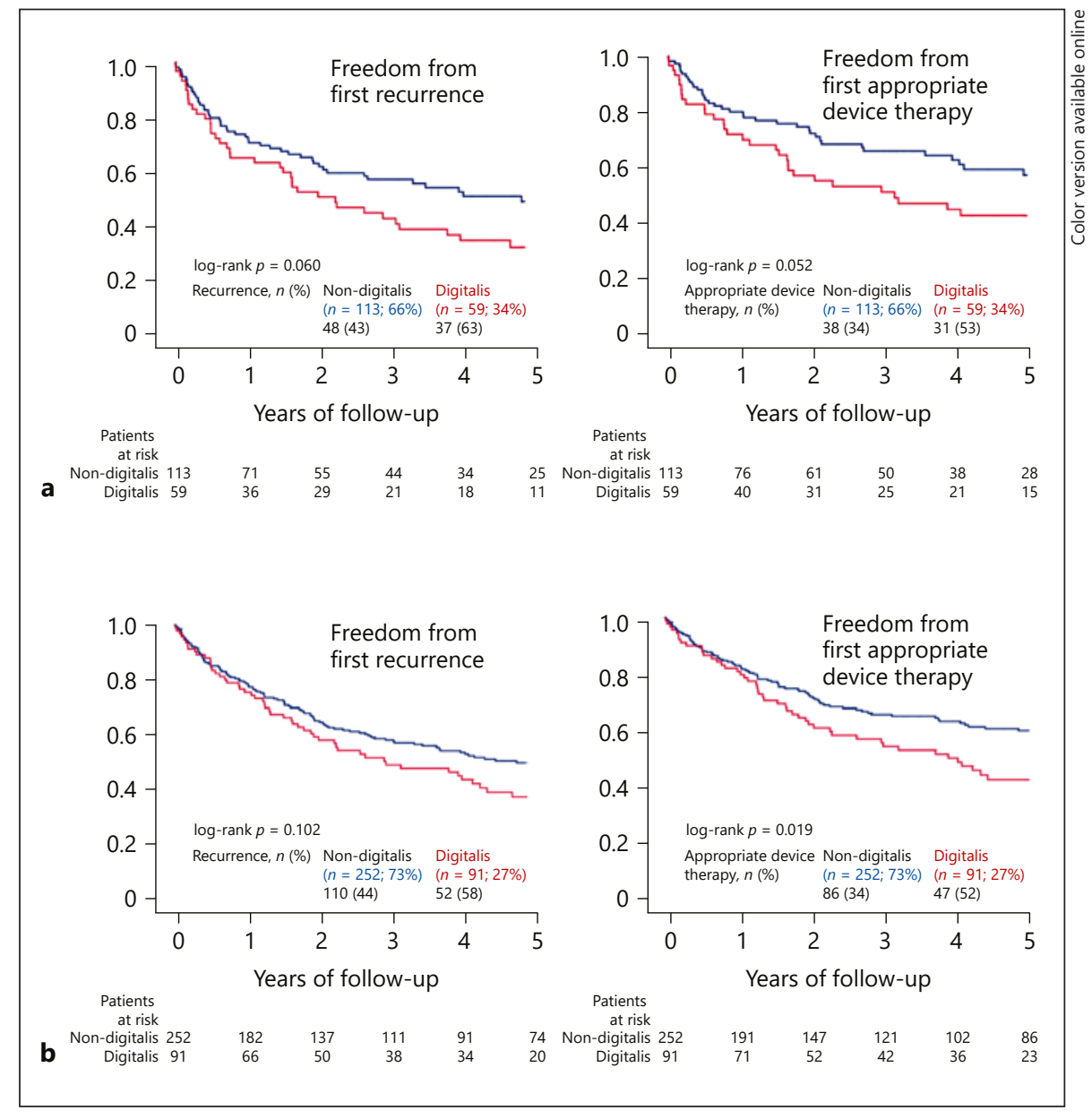

panel), whereas no difference was found for inappropriate device therapies (Fig. 2, right panel). The differences in first appropriate therapy were mainly attributable to first appropriate ATP therapy ( 31 vs. $18 \% ; p=0.007$ ). Overall appropriate ATP and shocks, as well as appropriate shocks per patient, were significantly higher among digitalis patients (Table 4).

Furthermore, digitalis patients had higher rates of overall first rehospitalization ( 38 vs. $21 \%$ ), mainly attributable to VT recurrences and acute $\mathrm{HF}$, as well as a higher rate of all-cause mortality at 5 years (33 vs. 20\%) (Table 4 ).

\section{Stratification by $A F$ and $H F$}

Among the AF patients $(n=172)$, digitalis therapy was associated with decreased freedom from recurrence of ventricular tachyarrhythmia (63 vs. $43 \%$; $\log$-rank $p=$ $0.060 ; \mathrm{HR}=1.505 ; 95 \%$ CI $0.980-2.311 ; p=0.062$, statistical trend) (Fig. 3a, left panel), as well as with decreased freedom from appropriate ICD therapy ( 53 vs. $34 \%$; log- rank $p=0.052 ; \mathrm{HR}=1.594 ; 95 \%$ CI $0.992-2.561 ; p=$ 0.054 , statistical trend) (Fig. 3a, right panel). In the presence of "AF only" (without HF), digitalis was still associated with decreased freedom from recurrence of ventricular tachyarrhythmia (69 vs. $38 \%$; $\log$-rank $p=0.033$; $\mathrm{HR}=2.434 ; 95 \%$ CI $1.048-5.650 ; p=0.038)$ and ICD therapy (61 vs. $32 \%$; log-rank $p=0.043$; HR $=2.452 ; 95 \% \mathrm{CI}$ $0.099-6.017 ; p=0.050)$.

Among the HF patients $(n=343)$, digitalis was not associated with decreased freedom from recurrence (logrank $p=0.102$ ) (Fig. 3b, left panel), but with decreased freedom from appropriate device therapy (52 vs. 34\%; $\log$-rank $p=0.019 ; \mathrm{HR}=1.527 ; 95 \%$ CI 1.070-2.178; $p=$ 0.020) (Fig. 3b, right panel).

Since the HF group still comprised more patients with concomitant AF, the impact of digitalis was reevaluated after having excluded the AF patients from the HF subgroup. In "HF-only" patients (without AF) $(n=222)$, digitalis was associated with comparable rates of recurrent 


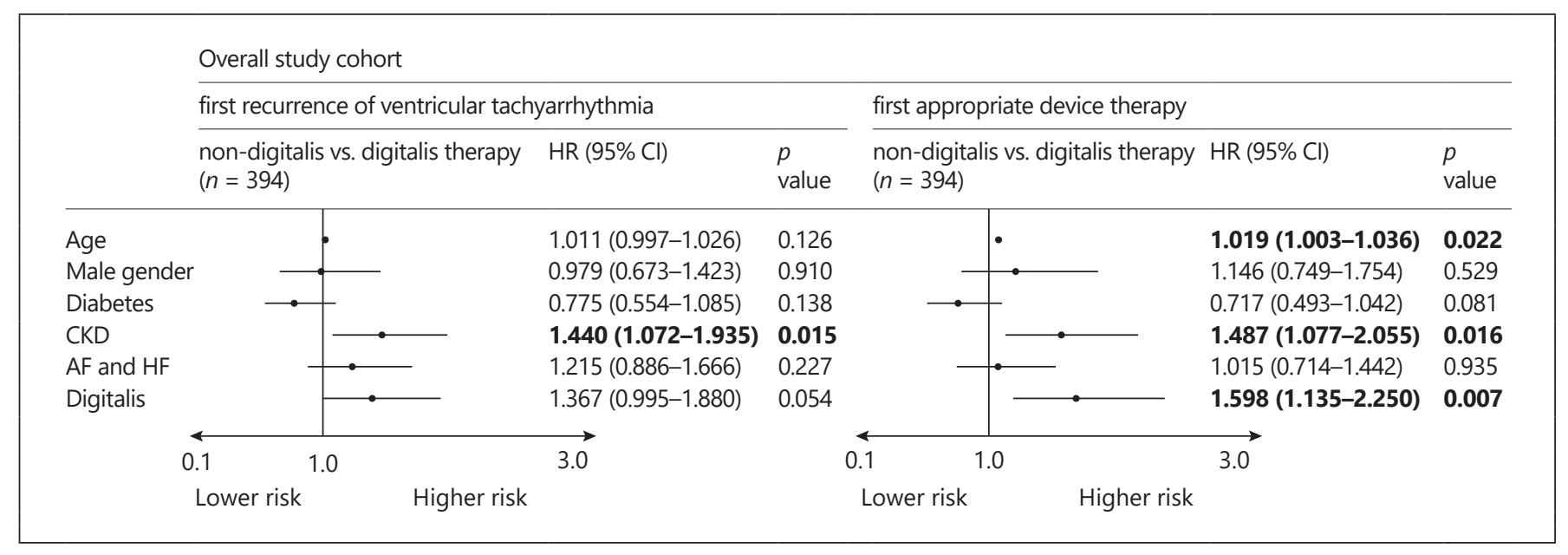

Fig. 4. Digitalis therapy was still associated with an increased risk of recurrent VT/VF within the entire study cohort (left columns) and appropriate ICD therapy (right columns) after multivariable adjustment in Cox regression models. Bold denotes significance. AF, atrial fibrillation; HF, heart failure; VT, ventricular tachycardia; VF, ventricular fibrillation; ICD, implantable cardioverter defibrillator; $\mathrm{CKD}$, chronic kidney disease.

ventricular tachyarrhythmia (53 vs. $43 \%$; $\log$-rank $p=$ 0.362 ), whereas higher rates of appropriate ICD therapy were still observed (53 vs. $34 \%$; $\log$-rank $p=0.053$; HR = 1.590; 95\% CI $0.990-2.553 ; p=0.055$, statistical trend) (data not shown).

\section{Multivariable Cox Regression Models}

Even after multivariable adjustment, digitalis treatment was still associated with a 1.4-fold higher risk of first recurrence of ventricular tachyarrhythmia $(\mathrm{HR}=1.367$; 95\% CI 0.995-1.880; $p=0.054$ ) (Fig. 4, left panel), which was still proven especially for ICD recipients for secondary prevention $(\mathrm{HR}=1.588 ; 95 \% \mathrm{CI}=1.053-2.394 ; p=$ 0.027 ) (data not shown). Accordingly, digitalis treatment was still associated with a 1.6-fold higher risk of appropriate device therapy $(\mathrm{HR}=1.598$; 95\% CI 1.135-2.250; $p=$ 0.007) (Fig. 4, right panel).

\section{Discussion}

The present study evaluated the prognostic impact of digitalis treatment on the primary endpoint of recurrence of ventricular tachyarrhythmia, as well as on secondary endpoints, such as appropriate ICD therapy, rehospitalization, and all-cause mortality at 5 years of follow-up, in ICD recipients with HF and AF surviving index episodes of ventricular tachyarrhythmia with additional betablocker treatment.

Impact of Digitalis on Recurrent

Ventricular Tachyarrhythmias
This study suggests that digitalis treatment (digoxin in $68 \%$ and digitoxin in $32 \%$ of the cases) decreases freedom from first recurrent ventricular tachyarrhythmia and from first appropriate device-related therapy. Digitalis especially increased the rates of recurrent sustained VT and of appropriate ATP therapy. Furthermore, negative impacts of digitalis, especially increased rates of appropriate ICD therapy, were seen in patients with HF only and in those with AF only. The adverse prognostic impact of digitalis was also seen after multivariable adjustment. Furthermore, patients treated with digitalis had a higher rate of rehospitalization and all-cause mortality at 5 years.

Focusing on current trends in pharmacotherapeutics, a decline in prescriptions of digoxin was demonstrated within an observational registry including more than 250,000 patients with HF with a reduced ejection fraction (HFrEF) and those with a preserved ejection fraction from 2005 until 2014 [17]. The recommendation to treat patients with digitalis is based on recommendation class II with a level of evidence B, as recently stated within European guidelines, for patients with symptomatic HFrEF to reduce the number of HF-related rehospitalizations [11]. In contrast, digitalis is recommended for patients with AF to control tachycardiac episodes irrespective of the presence of systolic HF (class I recommendation, level of evidence B) [12].

These heterogeneous recommendations for the use of digitalis, especially in HF, are based on recent studies, which demonstrated increasing rates of mortality [15, 24-30]. 
Although the prognostic impact of digitalis on all-cause mortality has been analyzed in many studies with different findings, there are only few studies that focus on the development of recurrence of ventricular tachyarrhythmia, especially disregarding ICD recipients after they had already survived index episodes of ventricular tachyarrhythmia. Therefore, the present study adds to the scientific evidence about the use of digitalis, since it was demonstrated that digitalis treatment of ICD recipients with HF or AF was especially associated with decreased freedom from appropriate ICD therapy, which was demonstrated in AF and HF patients (even after excluding AF patients). These data therefore support the evidence for a careful use of digitalis in patients surviving ventricular tachyarrhythmias despite the presence of an activated ICD.

Digoxin was evaluated in 350 consecutive HF patients with LVEFs $<35 \%$ and an activated CRT-D for primary prevention, where it was associated with an increased risk of appropriate shock therapy [19]. Accordingly, the MADIT-CRT trial demonstrated an increased risk of fast VT and VF [18]. Furthermore, Mina et al. [20] retrospectively analyzed 202 patients with either AF, HF (LVEF $<45 \%)$, or both and observed increased rates of ICD shock, electrical storm, and rehospitalization among 55 patients treated with digoxin at the 1-year follow-up. In contrast to prior ICD studies, the present one applies a different point of view, meaning a "secondary preventive effect" in a higher-risk population, in patients surviving at least one episode of VT. In these patients, treatment with digitalis increased the risk of recurrent episodes of ventricular tachyarrhythmia, appropriate device therapy, rehospitalization, and mortality.

However, besides treatment with digitalis, ventricular arrhythmogenicity is influenced by various factors. Especially AF represents an important predictor of ventricular tachyarrhythmia and SCD. An increased risk of tachyarrhythmia goes along with a higher incidence of shortlong cycles that can degenerate into VT among AF patients $[9,31,32]$. It may therefore be assumed that the increased risk of recurrence of ventricular tachyarrhythmia is triggered by the interaction of digitalis and $\mathrm{AF}$, rather than by digitalis therapy its own. Data from the Outcomes Registry for Better Informed Treatment of Atrial Fibrillation (ORBIT-AF) suggest that $62 \%$ of AF patients are symptomatic (European Heart Rhythm Association [EHRA] class $\geq 2$ ) and $17 \%$ even have severe symptoms (EHRA class 3-4), combined with a decreased quality of life [33]. However, the authors observed a comparable distribution of digoxin use between patients in EHRA class 1 and those in EHRA class $>1$. In contrast, a greater supply with digitalis was observed in symptomatic patients than in asymptomatic patients among 2,452 originally symptomatic AF patients at after 1 year of follow-up [34]. The authors of that study found increased risks of cardiac rehospitalization, stroke, and HF, worsening among patients with symptomatic AF. However, this study was not able to separate the interaction of AF symptoms with digitalis use, even when focusing on the subgroup of AF patients only, because there might have been patients with more severe AF symptoms in the digitalis group, which cannot be controlled for retrospectively.

To retrospectively control for some potential confounding due to different pharmacological interactions, all study patients were treated with beta-blockers, which guarantees further homogeneity beyond equally distributed comorbidities and comparability between the two therapeutic regimens. In particular, the European guidelines demand studies evaluating the combined treatment with beta-blockers and digitalis, especially for patients with HFrEF and AF [11]. The combination of beta-blockers and digoxin was analyzed by Lam et al. [35] using a propensity score-matched cohort of 334 patients, observing significantly lower rates of any hospital readmissions at 30 days; no differences in mortality rates were found in the digoxin group. In contrast, the present study found increased rates of rehospitalization especially due to ventricular tachyarrhythmia and acute HF despite betablocker treatment after long-term follow-up of 5 years.

In conclusion, this study demonstrates for the first time that treatment with digitalis is associated with an increased risk of - as well as a decrease in freedom from recurrent ventricular tachyarrhythmias among ICD recipients with $\mathrm{HF}$ and/or AF and additional beta-blocker therapy. An increased risk of appropriate ICD therapy was especially evident with secondary preventive ICDs. Digitalis treatment was moreover associated with decreased freedom from appropriate ICD therapy among AF patients and HF patients, even after excluding AF patients.

However, besides digitalis therapy itself, the interaction of digitalis and AF may be an important trigger of recurrences of ventricular tachyarrhythmia. Further studies or even randomized controlled trials are necessary to separate the ways of interaction of digitalis therapy and AF or HF with low LVEF.

\section{Study Limitations}

This observational and retrospective registry-based analysis reflects a realistic consecutive picture of the healthcare supply of high-risk patients presenting with 
ventricular tachyarrhythmias. The lost-to-follow-up rate regarding the evaluated endpoint of all-cause mortality was minimal. To minimize the lost-to-follow-up rate, all patients not presenting to the ICD follow-up at least one time after discharge were excluded from the analysis. The pharmacological therapies were based on the discharge medication at the index event. Despite multivariable adjustment, a certain prognostic impact of comorbidities in the digitalis group may not be excluded. All clinical data were documented reliably during routine clinical care by individual cardiologists being blinded to the final analyses, obviating the use of an independent clinical event committee. A selection bias due to the inhomogeneous distribution of AF rates may not be excluded, since the risk of recurrent ventricular tachyarrhythmias might be triggered by the interaction of digitalis and AF besides digitalis therapy itself. Moreover, the choice of treating patients with digitalis is made by physicians during routine clinical care. Unmeasured confounding (including the degree of AF or HF symptoms, i.e., NYHA or EHRA class, as well as important biomarkers, i.e., BNP) may also have affected our results, which may predominantly have occurred in digitalis patients. The present results need to be reevaluated within even more extensive and more representative multicenter registry data or even randomized controlled trials, especially focusing on the effect of digitalis on AF patients, controlling for confounding AF symptoms.

\section{Statement of Ethics}

This study was carried out according to the principles of the Declaration of Helsinki and was approved by the Medical Ethics Committee II of the Medical Faculty Mannheim, Heidelberg University, Germany.

\section{Disclosure Statement}

The authors declare that they do not have any conflict of interest.

\section{References}

1 Moss AJ, Hall WJ, Cannom DS, Daubert JP, Higgins SL, Klein H, et al.; Multicenter Automatic Defibrillator Implantation Trial Investigators. Improved survival with an implanted defibrillator in patients with coronary disease at high risk for ventricular arrhythmia. N Engl J Med. 1996 Dec;335 (26):1933-40.

2 Antiarrhythmics versus Implantable Defibrillators (AVID) Investigators. A comparison of antiarrhythmic-drug therapy with implantable defibrillators in patients resuscitated from near-fatal ventricular arrhythmias. N Engl J Med. 1997 Nov;337(22): 1576-83.

3 John RM, Tedrow UB, Koplan BA, Albert CM, Epstein LM, Sweeney MO, et al. Ventricular arrhythmias and sudden cardiac death. Lancet. 2012 Oct;380(9852):1520-9.

4 Priori SG, Blomström-Lundqvist C, Mazzanti A, Blom N, Borggrefe M, Camm J, et al.; ESC Scientific Document Group. 2015 ESC Guidelines for the management of patients with ventricular arrhythmias and the prevention of sudden cardiac death: The Task Force for the Management of Patients with Ventricular Arrhythmias and the Prevention of Sudden Cardiac Death of the European Society of Cardiology (ESC). Endorsed by: Association for European Paediatric and Congenital Cardiology (AEPC). Eur Heart J. 2015 Nov;36(41): 2793-867.

5 Dorian P. Combination ICD and drug treatments-best options. Resuscitation. 2000 Aug; 45(3):S3-6.
6 McElwee SK, Velasco A, Doppalapudi H. Mechanisms of sudden cardiac death. J Nucl Cardiol. 2016 Dec;23(6):1368-79.

7 Rattanawong P, Upala S, Riangwiwat T, Jaruvongvanich V, Sanguankeo A, Vutthikraivit $\mathrm{W}$, et al. Atrial fibrillation is associated with sudden cardiac death: a systematic review and meta-analysis. J Interv Card Electrophysiol. 2018 Mar;51(2):91-104.

8 Chao TF, Liu CJ, Tuan TC, Chen SJ, Chen TJ, Lip GY, et al. Risk and Prediction of Sudden Cardiac Death and Ventricular Arrhythmias for Patients with Atrial Fibrillation - A Nationwide Cohort Study. Sci Rep. 2017 Apr; 7(1):46445.

9 Chen LY, Benditt DG, Alonso A. Atrial fibrillation and its association with sudden cardiac death. Circ J. 2014;78(11):2588-93.

10 Masarone D, Limongelli G, Rubino M, Valente F, Vastarella R, Ammendola E, et al. Management of Arrhythmias in Heart Failure. J Cardiovasc Dev Dis. 2017 Feb;4(1): E3.

11 Ponikowski P, Voors AA, Anker SD, Bueno $\mathrm{H}$, Cleland JG, Coats AJ, et al.; ESC Scientific Document Group. 2016 ESC Guidelines for the diagnosis and treatment of acute and chronic heart failure: the Task Force for the diagnosis and treatment of acute and chronic heart failure of the European Society of Cardiology (ESC)Developed with the special contribution of the Heart Failure Association (HFA) of the ESC. Eur Heart J. 2016 Jul; 37(27):2129-200.
12 Kirchhof P, Benussi S, Kotecha D, Ahlsson A Atar D, Casadei B, et al.; ESC Scientific Document Group. 2016 ESC Guidelines for the management of atrial fibrillation developed in collaboration with EACTS. Eur Heart J. 2016 Oct;37(38):2893-962.

13 Digitalis Investigation Group. The effect of digoxin on mortality and morbidity in patients with heart failure. N Engl J Med. 1997 Feb;336(8):525-33.

14 Ziff OJ, Kotecha D. Digoxin: the good and the bad. Trends Cardiovasc Med. 2016 Oct;26(7): 585-95.

15 Vamos M, Erath JW, Hohnloser SH. Digoxinassociated mortality: a systematic review and meta-analysis of the literature. Eur Heart J. 2015 Jul;36(28):1831-8.

16 Erath JW, Vamos M, Hohnloser SH. Effects of digitalis on mortality in a large cohort of implantable cardioverter defibrillator recipients: results of a long-term follow-up study in 1020 patients. Eur Heart J Cardiovasc Pharmacother. $2016 \mathrm{Jul} ; 2(3): 168-74$

17 Patel N, Ju C, Macon C, Thadani U, Schulte PJ, Hernandez AF, et al. Temporal Trends of Digoxin Use in Patients Hospitalized With Heart Failure: Analysis From the American Heart Association Get With The GuidelinesHeart Failure Registry. JACC Heart Fail. 2016 May;4(5):348-56.

18 Lee AY, Kutyifa V, Ruwald MH, McNitt S, Polonsky B, Zareba W, et al. Digoxin therapy and associated clinical outcomes in the MADIT-CRT trial. Heart Rhythm. 2015 Sep; 12(9):2010-7.
Impact of Digitalis on Recurrent

Ventricular Tachyarrhythmias 
19 Adelstein E, Schwartzman D, Jain S, Bazaz R, Saba S. Effect of digoxin on shocks in cardiac resynchronization therapy-defibrillator patients with coronary artery disease. Am J Cardiol. 2014 Mar;113(6):970-5.

20 Mina GS, Acharya M, Shepherd T, Gobrial G, Tekeste M, Watti H, et al. Digoxin Is Associated With Increased Shock Events and Electrical Storms in Patients With Implantable Cardioverter Defibrillators. J Cardiovasc Pharmacol Ther. 2018 Mar;23(2):142-8.

21 Al-Khatib SM, Stevenson WG, Ackerman MJ, Bryant WJ, Callans DJ, Curtis AB, et al. 2017 AHA/ACC/HRS guideline for management of patients with ventricular arrhythmias and the prevention of sudden cardiac death: Executive summary: A Report of the American College of Cardiology/American Heart Association Task Force on Clinical Practice Guidelines and the Heart Rhythm Society. Heart Rhythm. 2018 Oct;15(10):e190-e252.

22 Deneke T, Israel CW, Krug J, Nentwich K, Müller P, Mügge A, et al. Indikationen zur Katheterablation bei ventrikulärer Tachykardie. Dtsch Med Wochenschr. 2013 Sep;138 (39):1952-6.

23 Dickstein K, Vardas PE, Auricchio A, Daubert JC, Linde C, McMurray J, et al.; ESC Committee for Practice Guidelines. 2010 Focused Update of ESC Guidelines on device therapy in heart failure: an update of the 2008 ESC Guidelines for the diagnosis and treatment of acute and chronic heart failure and the 2007 ESC Guidelines for cardiac and resynchronization therapy. Developed with the special contribution of the Heart Failure Association and the European Heart Rhythm Association. Europace. 2010 Nov;12(11):1526-36.
24 Ziff OJ, Lane DA, Samra M, Griffith M, Kirchhof P, Lip GY, et al. Safety and efficacy of digoxin: systematic review and meta-analysis of observational and controlled trial data. BMJ. 2015 Aug;351:h4451.

25 Hood WB Jr, Dans AL, Guyatt GH, Jaeschke R McMurray JJ. Digitalis for treatment of heart failure in patients in sinus rhythm. Cochrane Database Syst Rev. 2014 Apr;(4):CD002901.

26 Al-Khateeb M, Qureshi WT, Odeh R, Ahmed AM, Sakr S, Elshawi R, et al. The impact of digoxin on mortality in patients with chronic systolic heart failure: a propensity-matched cohort study. Int J Cardiol. 2017 Feb;228:214-8.

27 Ouyang AJ, Lv YN, Zhong HL, Wen JH, Wei $\mathrm{XH}$, Peng HW, et al. Meta-analysis of digoxin use and risk of mortality in patients with atrial fibrillation. Am J Cardiol. 2015 Apr;115(7): 901-6.

28 Washam JB, Stevens SR, Lokhnygina Y, Halperin JL, Breithardt G, Singer DE, et al.; ROCKET AF Steering Committee and Investigators. Digoxin use in patients with atrial fibrillation and adverse cardiovascular outcomes: a retrospective analysis of the Rivaroxaban Once Daily Oral Direct Factor Xa Inhibition Compared with Vitamin K Antagonism for Prevention of Stroke and Embolism Trial in Atrial Fibrillation (ROCKET AF). Lancet. 2015 Jun;385(9985):2363-70.

29 Freeman JV, Reynolds K, Fang M, Udaltsova N, Steimle A, Pomernacki NK, et al. Digoxin and risk of death in adults with atrial fibrillation: the ATRIA-CVRN study. Circ Arrhythm Electrophysiol. 2015 Feb;8(1):49-58.

30 Al-Zakwani I, Panduranga P, Zubaid M, Sulaiman K, Rashed WA, Alsheikh-Ali AA, et al. Impact of Digoxin on Mortality in Patients With Atrial Fibrillation Stratified by Heart Failure: Findings From Gulf Survey of Atrial Fibrillation Events in the Middle East. J Cardiovasc Pharmacol Ther. 2016 May;21(3): 273-9.
31 Chang IC, Agamawi YM, Austin E, Adkisson WO, Roukoz H, von Wald LN, et al. Usefulness of Atrial Fibrillation as a Marker for Adverse Cardiovascular Outcomes in Both Primary and Secondary Prevention in Patients With Implantable Cardioverter-Defibrillators. Am J Cardiol. 2016 Nov;118(10):1497502.

32 Deneke T, Lawo T, Reinecke J, Buttler C, Grewe PH, Shin DI, et al. Predictors of sustained ventricular arrhythmia episodes in patients with primary ICD indication: male gender and AF in primary ICD prophylaxis. Herzschrittmacherther Elektrophysiol. 2011 Dec;22(4):219-25.

33 Freeman JV, Simon DN, Go AS, Spertus J, Fonarow GC, Gersh BJ, et al.; Outcomes Registry for Better Informed Treatment of Atrial Fibrillation (ORBIT-AF) Investigators and Patients. Association Between Atrial Fibrillation Symptoms, Quality of Life, and Patient Outcomes: Results From the Outcomes Registry for Better Informed Treatment of Atrial Fibrillation (ORBITAF). Circ Cardiovasc Qual Outcomes. 2015 Jul;8(4):393-402.

34 Guerra F, Brambatti M, Nieuwlaat R, Marcucci M, Dudink E, Crijns H, et al. Symptomatic atrial fibrillation and risk of cardiovascular events: data from the Euro Heart Survey. Europace. 2017 Dec;19(12):1922-9.

35 Lam PH, Bhyan P, Arundel C, Dooley DJ, Sheriff HM, Mohammed SF, et al. Digoxin use and lower risk of 30-day all-cause readmission in older patients with heart failure and reduced ejection fraction receiving $\beta$-blockers. Clin Cardiol. 2018 Mar;41(3): 406-12. 